IAU Colloquium 190 on Magnetic Cataclysmic Variables

ASP Conference Series, Vol. 315, 2004

Sonja Vrieimann 60 Mark Cropper, eds.

\title{
Magnetic Field Topology of Accreting White Dwarfs
}

\author{
Klaus Reinsch, Fabian Euchner, Klaus Beuermann \\ Universitäts-Sternwarte, Geismarlandstr. 11, 37083 Göttingen, Germany \\ Stefan Jordan \\ Institut für Astronomie und Astrophysik, Eberhard-Karls-Universität \\ Tübingen, Sand 1,72076 Tübingen, Germany
}

\begin{abstract}
We report first results of our systematic investigation of the magnetic field structure of rotating single magnetic white dwarfs and of white dwarfs in magnetic cataclysmic variables. The global magnetic field distributions on the isolated white dwarf HE 1045-0908 and the accreting white dwarfs in EF Eri and CP Tuc have been derived from phase-resolved flux and polarization spectra obtained with FORS1 at the ESO VLT using the systematic method of Zeeman tomography.
\end{abstract}

\section{Introduction}

Magnetic fields have been detected in $\sim 5 \%$ of the 2300 known single white dwarfs with field strengths ranging from $\sim 0.1-1000 \mathrm{MG}$ and peaking around $16 \mathrm{MG}$ (McCook \& Sion 1999, Wickramasinghe \& Ferrario 2000). A similar fraction of magnetic white dwarfs has been found in accreting close binary systems (cataclysmic variables), possibly with a more restricted range of field strengths (7-230 MG, Beuermann 1998). The origin of the magnetic fields is not well understood. The magnetic fluxes, which should be conserved during stellar evolution, are similar to those of the magnetic main sequence stars such as the Ap and Bp stars which constitute $\sim 5 \%$ of the normal main sequence stars. The decay times of the lowest multipole components are predicted to be long compared to the evolutionary ages of the white dwarfs. The magnetic field topologies, at least of isolated white dwarfs, are, therefore, likely to be relics of previous evolutionary phases. In accreting systems, the field structure in the outer layers of the white dwarf, however, may have been significantly changed if the accretion rate is high enough that accretion occurs more rapidly than ohmic diffusion (Cumming 2002).

Observational evidence suggests that the field topology of isolated and accreting white dwarfs can deviate drastically from that of a centered dipole (e.g. Wickramasinghe \& Ferrario 2000, Schwope 1995). Information about the strength, orientation, and structure of the surface magnetic field of white dwarfs so far has been mainly derived from the analysis of photospheric Zeeman absorption lines and thermally broadened cyclotron harmonics from the polar regions of accreting white dwarfs. The interpretation of Zeeman and cyclotron intensity 
spectra alone is, however, often ambiguous and circular polarization spectra are required to investigate the magnetic field structure in detail.

\section{Zeeman tomography}

We have developed a systematic method, called Zeeman tomography, to derive the global magnetic field distribution on rotating white dwarfs (Euchner et al. 2002). At present, it utilizes a database of 46800 sets of flux and circular polarization Zeeman spectra of homogeneous magnetic white dwarf atmospheres calculated with a code developed by S. Jordan for a broad range of field strengths $|\boldsymbol{B}|=1-400 \mathrm{MG}$, effective atmospheric temperatures $T=8000-50000 \mathrm{~K}$, and 9 field directions $\psi$ relative to the line of sight. All spectra are calculated for a surface gravity $\log g=8$ and use a simple limb darkening law which is independent of wavelength. The magnetic field structure is approximated by a multipole expansion of the scalar magnetic potential, using spherical harmonics with coefficients $l=1, \ldots, 5$ and $m=0$ for the zonal and sectoral periodicity of the field. The database models are integrated for the $\boldsymbol{B}$ distribution of 900 elements of the visible white dwarf surface at a given rotational phase. A least-squares optimization code based on an evolutionary strategy is used to reconstruct the multipole parameters from a set of flux and polarization spectra obtained at different rotational phases of the white dwarf.

The Zeeman tomography method has been thoroughly tested with the reconstruction of various field geometries from synthetic spectra (Euchner et al. 2002). Here, we present first results of our application to real observational data.

\section{Observations}

We have obtained spin-phase resolved circular spectropolarimetry of a sample of isolated and accreting magnetic white dwarfs using FORS1 at the ESO VLT during four observing runs between May 1999 and December 2000. The instrument has been set up with a Wollaston prism and a rotatable quarter-wave retarder plate. Exposures have been alternately taken with retarder plate position angles $\phi=-45^{\circ}$ and $\phi=+45^{\circ}$ in order to correct for instrumental polarization effects and linear-polarization cross-talk during the data reduction. Spectra of the target star and comparison stars in the field have been obtained simultaneously by using the multi-object spectroscopy mode of FORS. This allowed us to derive individual correction functions for the atmospheric absorption losses in the target spectra and to check the data for remnant instrumental polarization. The target spectra cover the wavelength range $3800-7900 \AA$ at a spectral resolution $\lambda / \Delta \lambda=440$. A signal-to-noise ratio $S / N \sim 100$ has been reached for the individual flux spectra at a spin-phase resolution $\Delta \Phi \sim 0.2$. All raw data have been reduced using the context MOS of the ESO MIDAS package.

All our spectropolarimetric observations of magnetic CVs have been obtained while the systems were in a low-state of accretion. Hence, the Zeemansplit photospheric hydrogen absorption lines were clearly visible as the emission of the white dwarf was not diluted by emission from the accretion stream. 


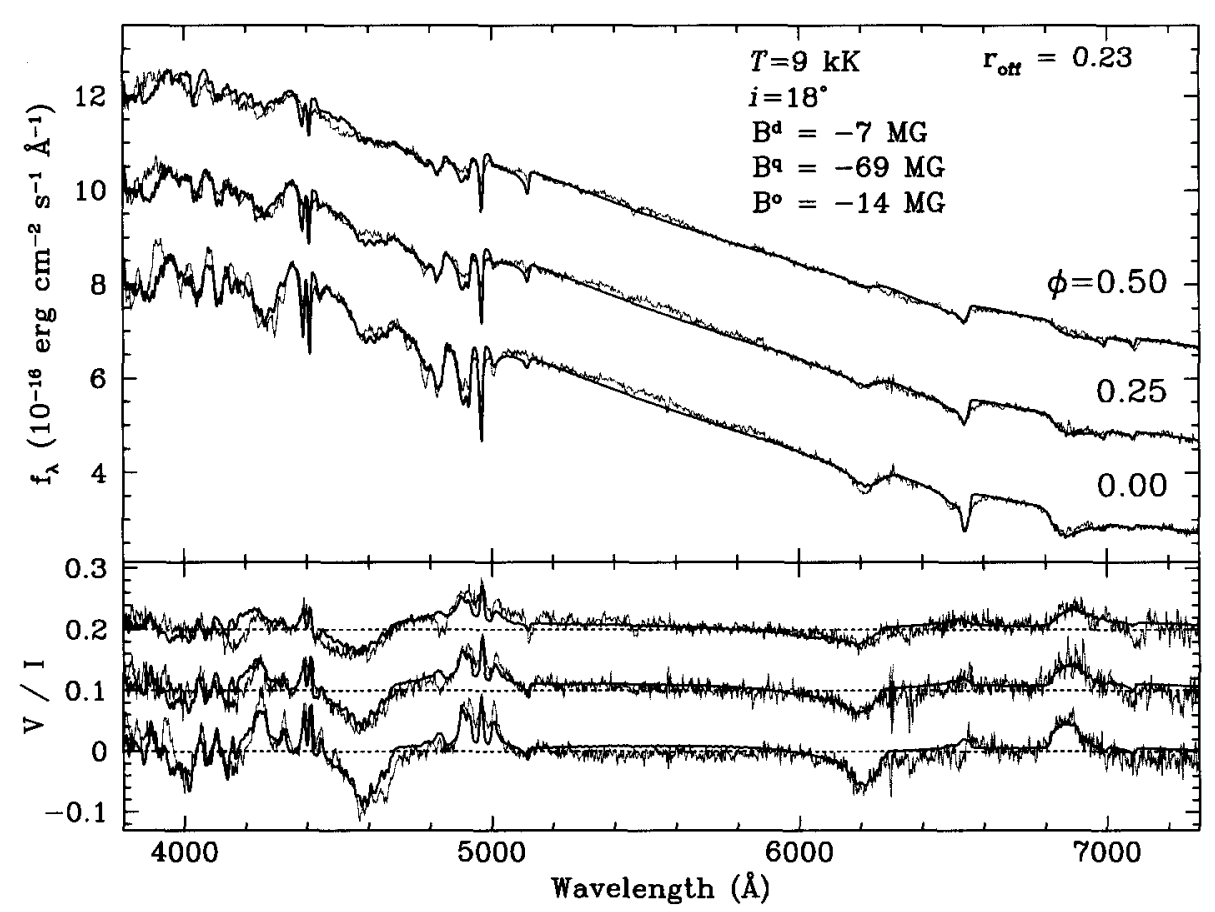

Figure 1. Flux (top) and circular polarization (bottom) spectra of HE 1045-0908 at (the arbitrary) rotational phases $\Phi=0.0,0.25$, and 0.5 . The synthetic spectra for the best-fit model (thick line) consisting of a dipole, quadrupole, and octopole field component is shown superimposed on the observed spectra (grey curve). For clarity, the upper two flux spectra have been offset by 2 and 4 flux units, respectively, and the polarization spectra by 0.1 and 0.2 units, respectively.

\section{Results}

\subsection{HE 1045-0908}

HE 1045-0908 is an isolated hydrogen-line white dwarf with a polar field strength $B \sim 20 \mathrm{MG}$ and a rotational period $P_{\text {rot }} \sim 2-4 \mathrm{~h}$ (Reimers et al. 1994, Schmidt et al. 2001). Our observations cover $\sim 2 \mathrm{~h}$ during which drastic variability in the flux and polarization spectra has been seen (Fig. 1). The field topology can be described by a combination of aligned dipole, quadrupole, and octopole terms with a common offset perpendicular to the magnetic axis (Fig. 2). An almost equally well fit has been obtained for a multipole expansion including the zonal components up to $l=5$. It is reassuring that both fits yield similar ranges for the observed field strengths and viewing angles.

\subsection{EF Eri}

Spectropolarimetric observations of EF Eri have been obtained during two epochs, in December 1999 and in November 2000 and cover in total 2 spin cycles of the 


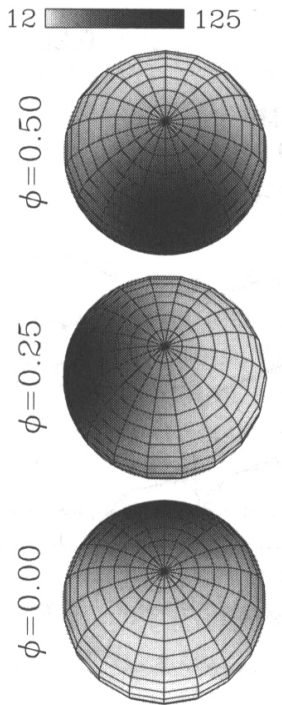

B / MG
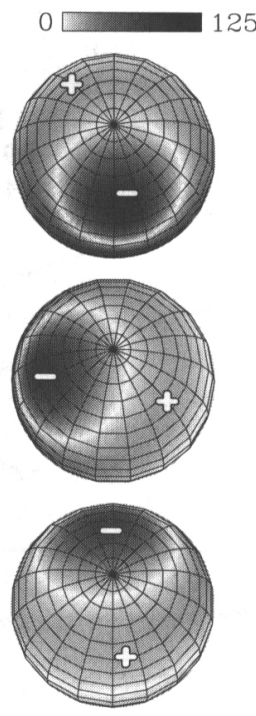

$\left|\mathrm{B}_{1}\right| / \mathrm{MG}$ filling factor: 0 ma.128

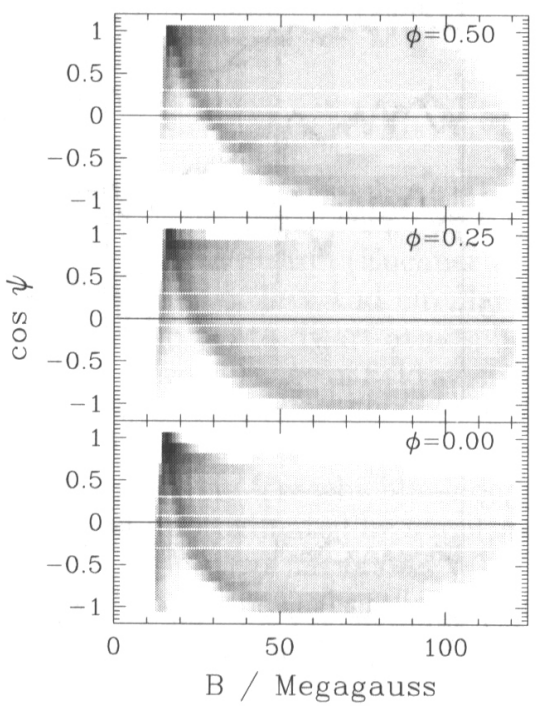

Figure 2. Distribution of the total magnetic field strength $B$ and its longitudinal component $B_{1}$ on the white dwarf in HE 1045-0908 at (arbitrary) rotational phases $\Phi=0.0,0.25$, and 0.5 (left) and frequency distribution of $B$ and the viewing direction $\operatorname{cosine} \cos \Phi$ (right).

white dwarf. Rotational phases have been calculated using the ephemeris given by Piirola et al. (1987), with $\Phi=0.0$ corresponding to the narrow minimum in the infrared light curve of EF Eri, i.e. to the phase when the magnetic pole is closest to the observer. As our data show little variation in the Zeeman features modeling has been done for the phase averaged flux and polarization spectra (Fig. 3 and 4). The data are sufficiently well described by a multipole expansion including the zonal components up to $l=5$. We note, however, that there are still systematic residuals in our best fit solution which indicate that the field topology must be even more complex.

\subsection{CP Tuc}

CP Tuc (= AX J2315-592) has been discovered by Thomas \& Reinsch (1996) who derived an upper limit of the magnetic field strength at the accreting pole $B \leq 17 \mathrm{MG}$ from the properties of the optically thin cyclotron emission. Our spectropolarimetric observations cover $\sim 0.5$ spin periods of CP Tuc (Fig. 5 and 6 ). Rotational phases have been calculated using the ephemeris given by Ramsay et al. (1999) where $\Phi=0.0$ is defined by the minimum of the X-ray light curve. Our best-fit solution for the field topology comprises a dipole, quadrupole plus octopole combination. Again, residual features indicate an even more complex field. The high polar field strength found here is not contradicting the much lower upper limit derived by Thomas \& Reinsch (1996) for the field strength in the accretion region as this region is probably offset from the magnetic poles. 


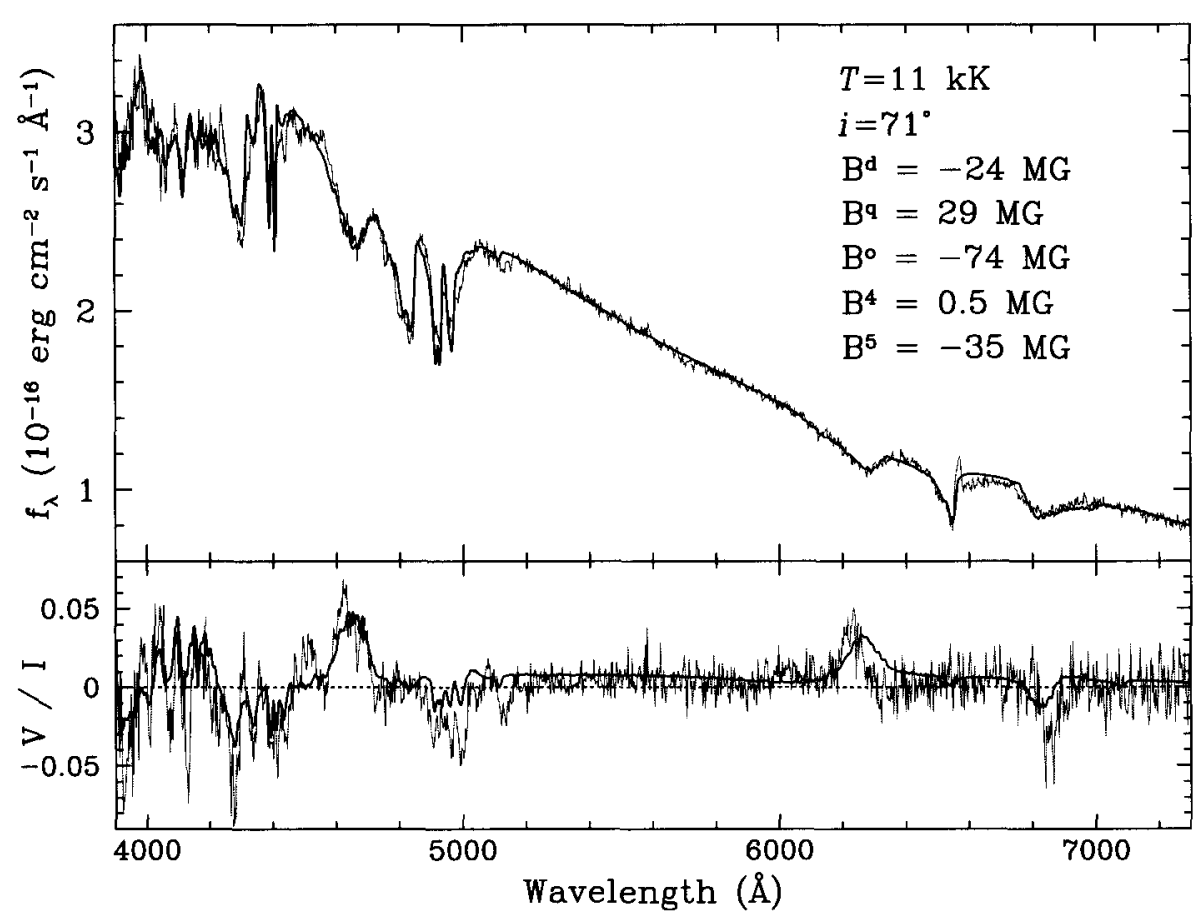

Figure 3. Average flux (top) and circular polarization (bottom) spectrum of EF Eri. The synthetic spectrum for the best-fit model (thick line) consisting of a five component multipole expansion is shown superimposed on the observed spectrum (grey curve).

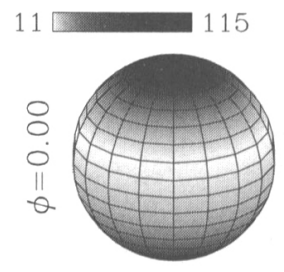

$\mathrm{B} / \mathrm{MG}$
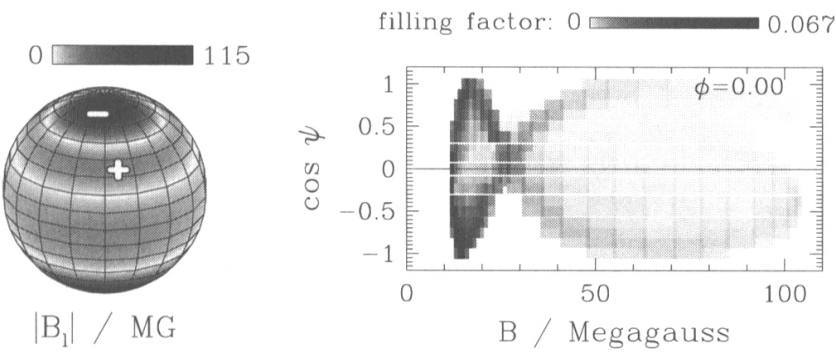

Figure 4. Distribution of the total magnetic field strength $B$ and its longitudinal component $B_{1}$ on the white dwarf in EF Eri at spin phase $\Phi=0.0$ (left) and frequency distribution of $B$ and the viewing direction cosine $\cos \Phi$ (right). 


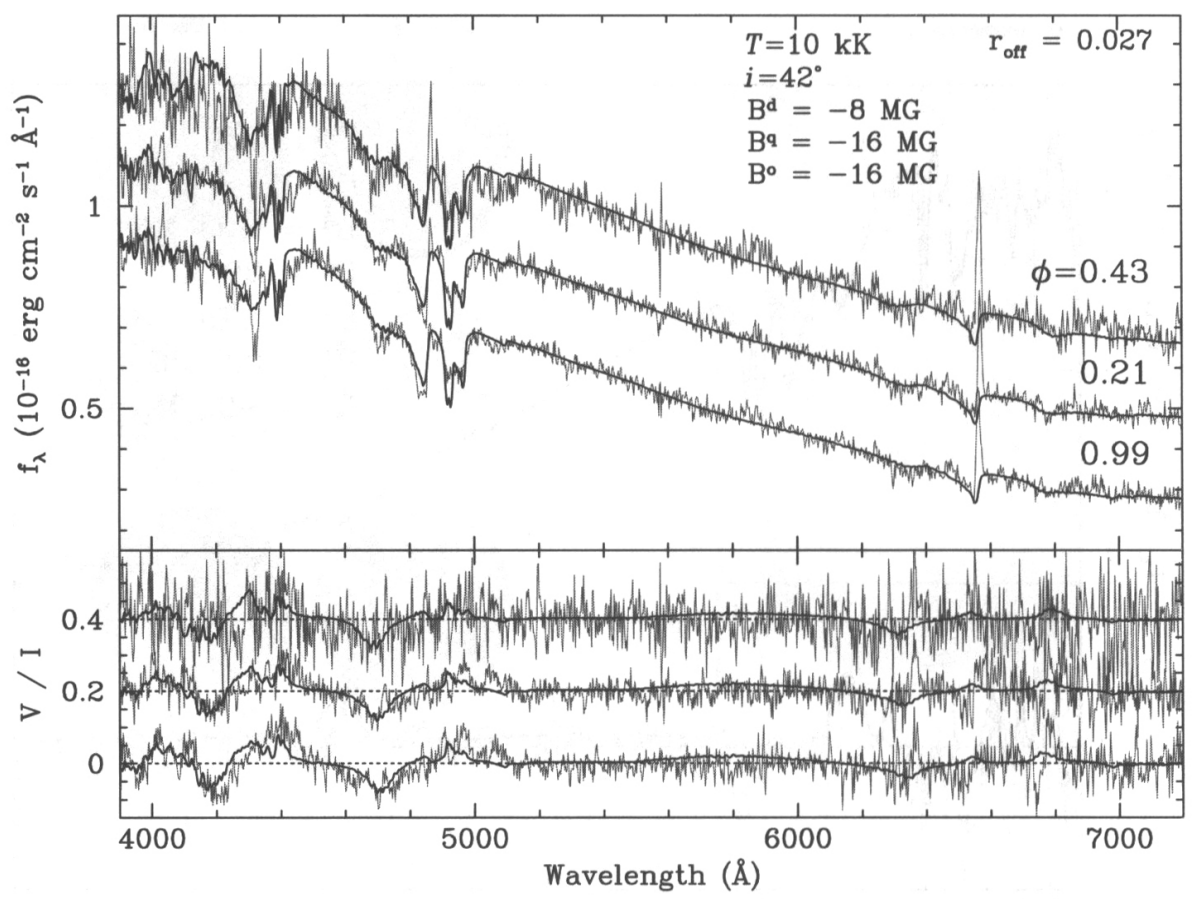

Figure 5. Flux (top) and circular polarization (bottom) spectra of CP Tuc at spin phases $\Phi=0.99,0.21$, and 0.43 . The synthetic spectra for the best-fit model (thick line) consisting of a dipole, quadrupole, and octopole field component is shown superimposed on the observed spectra (grey curve). The upper two flux and polarization spectra have been offset by 0.2 and 0.4 units, respectively.

\section{Conclusions}

We have shown that Zeeman tomography is a suitable systematic method to derive the global magnetic field distribution on rotating white dwarfs. With this method we have obtained for the first time detailed information about the range of field strengths and the field topology of a sample of isolated and accreting white dwarfs. Our results clearly demonstrate that a single value as obtained with hitherto available methods is not sufficient to quantify the field of magnetic white dwarfs. Overall our model fits are in excellent agreement with observations. Remaining differences indicate that the field topology is even more complex than described by an up to 5 component multipole expansion. A more detailed discussion of our results will be presented elsewhere (Euchner et al., in prep.).

Acknowledgments. Based on observations collected at the European Southern Observatory, Chile under program numbers 63.P-0003(A), 64.P-0150(C), and 66.D-0128(A). This work was supported in part by BMBF/DLR grant 50 OR 99036. 

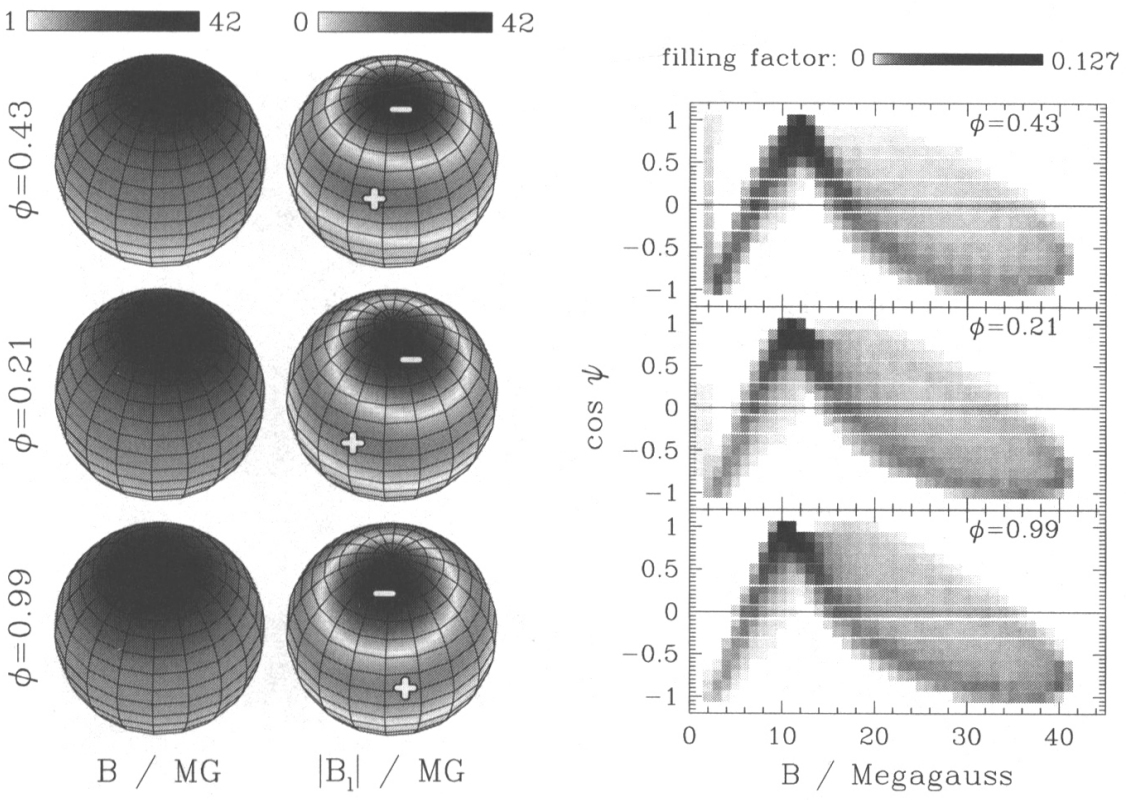

Figure 6. Distribution of the total magnetic field strength $B$ and its longitudinal component $B_{1}$ on the white dwarf in CP Tuc at rotational phases $\Phi=0.99,0.21$, and 0.43 (left) and frequency distribution of $B$ and the viewing direction cosine $\cos \Phi$ (right).

\section{References}

Beuermann, K. 1998, in High Energy Astronomy and Astrophysics, Tata Inst. of Fund. Res., 100

Cumming, A. 2002, MNRAS, 333, 589

Euchner, F., Jordan, S., Beuermann, K., Gänsicke, B.T., \& Hessman, F.V. 2002, A\&A, 390, 633

McCook, G.P. \& Sion, E.M. 1999, ApJS, 121, 1

Piirola, V., Coye, G.V., \& Reiz, A. 1987, A\&A186, 120

Ramsay, G., Potter, S.B., Buckley, D.A.H., \& Wheatley, P.J. 1999, MNRAS, 306,809

Reimers, D., Jordan, S., Köhler, T., \& Wisotzki, L. 1994, A\&A, 285, 995

Schmidt, G.D., Vennes, S., Wickramasinghe, D.T., \& Ferrario, L. 2001, MNRAS, 328,203

Schwope, A.D. 1995, Rev. Mod. Astron., 8, 125

Thomas, H.-C. \& Reinsch, K. 1996, A\&A, 315, L1

Wickramasinghe, D.T. \& Ferrario, L. 2000, PASP, 112, 873 